\title{
Coexistence and Coupling of Superconductivity and Magnetism in Thin Film Structures
}

\author{
A. J. Drew, ${ }^{1, *}$ S. L. Lee, ${ }^{1}$ D. Charalambous,${ }^{2}$ A. Potenza, ${ }^{3}$ C. Marrows,${ }^{3}$ H. Luetkens,${ }^{4, \dagger}$ A. Suter, ${ }^{4}$ T. Prokscha, ${ }^{4}$ \\ R. Khasanov, ${ }^{4, \ddagger}$ E. Morenzoni, ${ }^{4}$ D. Ucko, ${ }^{5,8}$ and E. M. Forgan ${ }^{5}$ \\ ${ }^{1}$ School of Physics and Astronomy, University of St. Andrews, St. Andrews, KY16 9SS, Scotland, United Kingdom \\ ${ }^{2}$ Department of Physics, University of Lancaster, Lancaster, LA1 4YB, United Kingdom \\ ${ }^{3}$ School of Physics and Astronomy, University of Leeds, Leeds, LS2 9JT, United Kingdom \\ ${ }^{4}$ Labor für Myonspinspektroskopie, Paul Scherrer Institute, CH-5232 Villigen PSI, Switzerland \\ ${ }^{5}$ School of Physics and Astronomy, University of Birmingham, Birmingham, B15 2TT, United Kingdom
}

(Received 18 February 2005; published 1 November 2005)

\begin{abstract}
Superconducting and magnetic order are usually mutually exclusive, and are found to coexist in relatively few materials. We have obtained direct evidence for a spin-density wave (SDW) coexisting with bulk superconductivity in a ferromagnetic-superconducting trilayer. In the superconducting state the amplitude of the SDW is enhanced and modeling the data also suggests a $\pi / 2$ phase shift of one component of the SDW, implying a profound coupling of these two forms of order.
\end{abstract}

In conventional $s$-wave superconductors the conduction electrons form Cooper pairs, in which the electronic spins are oppositely aligned to form a spin-singlet state. The presence of a magnetic impurity in such a system gives rise to an exchange field that will tend to align the spins of the surrounding electrons parallel to it. Thus in order to explain the robustness of superconducting order to the presence of magnetic species, more exotic scenarios may have to be invoked, for example, spin-triplet $p$-wave superconductivity. In a contrasting scenario, one considers the spin-singlet state to persist, and the potential energy associated with the exchange field causes each of the two electrons to have different kinetic energy. The resultant linear momentum of the pair leads to a spatial oscillation of the superconducting wave function, which periodically reverses sign [1,2]. This Larkin-Ovchinnikov-FuldeFerrell (LOFF) state is thus an $s$-wave spin-singlet system but possesses nonzero linear momentum, and is therefore distinct from either a $p$-wave or a conventional $s$-wave superconductor. It is thus of interest to study systems in which magnetism and superconductivity coexist, in order to probe exotic ground states.

Artificially fabricated multilayered thin-film structures, where superconducting (S) and ferromagnetic (FM) materials are juxtaposed in neighboring layers, are an ideal medium in which to explore the interaction of the two order parameters at the interface. For a normal metal (N)/FM multilayer, the exchange field can cause a periodic oscillation of the electron-spin density inside the normal layer. The period of this spin-density wave (SDW) is determined by an enhancement of the wave-vectordependent susceptibility at extremal spanning vectors of the Fermi surface [3], as in the Ruderman-Kittel-KasuyaYosida (RKKY) interaction [4,5]. In contrast, for the case of a FM layer that is bounded on both sides by $\mathrm{S}$ material, a LOFF-type scenario gives rise to a phase difference of the superconducting wave function across the layer $[6,7]$. The value of the phase difference will depend on the layer thickness compared to the wavelength of the spatial oscillation. While there are convincing reports of both spatial modulation of the superconducting order parameter inside the FM layer [8] and for the existence of a SDW inside a normal layer bounded by FM material [9], relatively little attention has been given to the related effect occurring inside a $S$ layer. Here we report such a measurement, where we demonstrate that remarkably a SDW may persist in, and couple to, the superconducting state.

The principal method of investigation is the recently developed low energy muon-spin rotation (LE- $\mu \mathrm{SR}$ ) technique [10]. This is analogous to conventional muon-spin rotation ( $\mu \mathrm{SR})$, with the additional advantage that with the LE- $\mu$ SR technique one can control the depth of implantation into the surface of a sample via control of the muon energy. The technique is ideal for the investigation of magnetism in thin films, where a typical thickness is of the order of a few hundred $\mathrm{nm}$. LE- $\mu$ SR has recently been used to measure the depth profile of the magnetic screening in a high-temperature superconductor thin film [11], to directly observe nonlocal superconductivity effects in $\mathrm{Pb}$ [12], and also to observe a SDW in Fe/Ag multilayers [9]. In our experiment, spin-polarized positive muons are brought to rest in the sample and their spins precess in the local internal flux density. The muon decays with a mean lifetime of $2.197 \mu \mathrm{s}\left(\mu^{+} \rightarrow e^{+}+\bar{\nu}_{\mu}+\nu_{e}\right)$, emitting positrons preferentially along the muon-spin direction; the positrons are detected using an array of scintillation detectors. By averaging over typically two million muon events, the temporal evolution of the muon-spin polarization direction may thus be determined [10].

The sample was an $\mathrm{Fe} / \mathrm{Pb} / \mathrm{Fe}$ trilayer with a Mo capping layer to inhibit oxidation. The dimensions were $20 \times$ $22 \mathrm{~mm}$, with $\mathrm{Fe}$ thickness 3.0(3) nm, Pb thickness 215(5) $\mathrm{nm}$, and Mo thickness 8.9(1) nm. It was grown on a high quality silicon wafer (100) substrate using dc sput- 
tering. The superconducting properties of the films were characterized using dc resistivity and magnetization measurements, yielding a superconducting transition temperature $T_{c}=5.8(1) \mathrm{K}$. Layer thicknesses were calibrated using ex situ $\mathrm{x}$-ray reflectometry and polarized neutron reflectometry (PNR), using the CRISP instrument at ISIS. By fitting an optical neutron reflectivity model [13] to the PNR data, we determined the thickness of the magnetic and nonmagnetic layers in our sample (shown schematically in the inset of Fig. 1) and its roughness. Using this information, it is possible to calculate the muon implantation depth distribution $p(z)$ for the relevant implantation energies, shown in Fig. 1, using a well-proven Monte Carlo algorithm [14].

Figure 2 shows examples of the time evolution of the muon-spin polarization, for an implantation energy of $10 \mathrm{keV}$. The measurements were made in a field of $20 \mathrm{mT}$ applied parallel to the film and perpendicular to the initial muon-spin polarization, at temperatures both above and below $T_{c}$. Since this precession signal is clearly nontrivial, more insight can be gained from the frequency domain data of Fig. 3, which shows the $p(B)$ derived from the time domain data via a maximum entropy Fourier transform technique $[15,16]$. Figure 3(a) shows the measured probability distributions of values of magnetic field $p(B)$ inside the sample in the normal metallic state at a temperature of $10 \mathrm{~K}$, for muon implantation energies corresponding to those shown in Fig. 1. The unusual $p(B) \mathrm{s}$ shown in Fig. 3(a) reflect a complicated flux density profile, arising from a SDW induced in the $\mathrm{Pb}$ layer by the exchange field from the Fe layers, as previously demonstrated for $\mathrm{Fe} / \mathrm{Ag} / \mathrm{Fe}$ trilayers [9]. The satellite peaks in the data are related to the turning points of the SDW [9]. The fraction of muons stopping in the Fe layer experience a very large field and precess too fast for any oscillating signal from them to be detected.

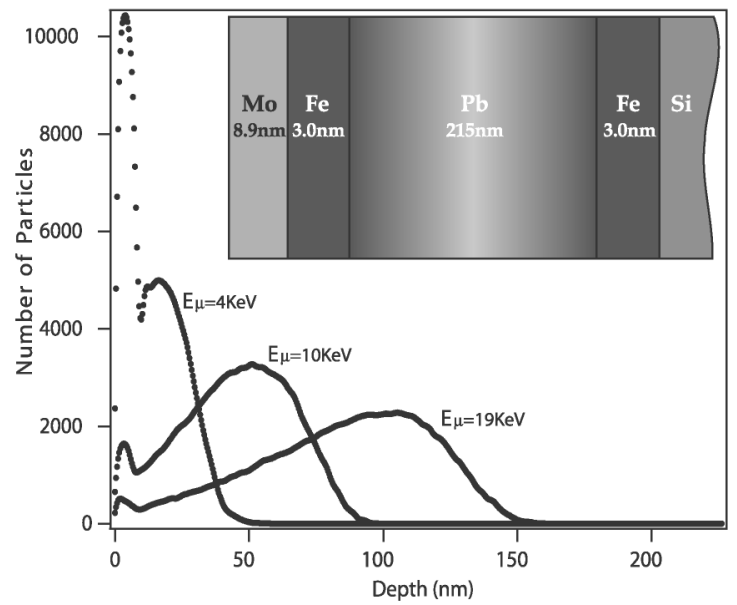

FIG. 1. The calculated implantation profile for muons stopping in the sample, plotted for different incident energies. Inset: the composition and structure of the sample, as determined by PNR.
The fits to our data in the frequency domain are achieved by nonlinear regression and are given in Fig. 3(a) by the shaded areas, and simply reflect the local depth-dependent magnetization due to the SDW. At a given temperature, an identical model is used for all energies, and any differences arise only from the muon stopping profile of Fig. 1. The magnetization within the $\mathrm{Pb}$ spacer layer is given by the addition of the magnetization, $M(z)$, induced in the $\mathrm{Pb}$, originating from each of the two $\mathrm{Fe} / \mathrm{Pb}$ interfaces. In our model, $M(z)$ is assumed to take the following form [3]:

$$
M(z)=\sum_{i} A_{i} \frac{\sin \left(2 k_{i} z+\phi_{i}\right)}{z^{n_{i}}},
$$

where $k_{i}$ are the wave vectors of the SDW, $z$ the distance into the spacer layer, and $A_{i}$ is an adjustable parameter for the coupling strength of the spin-density wave of wave vector $k_{i}$. Our polycrystalline $\mathrm{Pb}$ films grow preferentially with the [111] direction perpendicular to the surface of the film. The fits were found to converge to two wave vectors, $k_{1}=2.3(2) \mathrm{nm}^{-1}$ and $k_{2}=15.8(2) \mathrm{nm}^{-1}$. These values are in excellent agreement with those obtained from de Haas-van Alphen quantum oscillation measurements of $\mathrm{Pb}\left(2.31 \mathrm{~nm}^{-1}\right.$ and $\left.15.81 \mathrm{~nm}^{-1}\right)[17,18]$ and correspond to wave vectors that connect flat pieces of the Fermi surface along the (111) direction in reciprocal space. One additional complication, which has been accounted for in the model, arises from the aliasing effect due to the discrete sampling steps of the muons, which reside at fixed sites within the crystal lattice [9]. This has been incorporated using the known lattice parameters for $\mathrm{Pb}$, without any adjustment. On fixing the wave vectors to those found from the de Haas-van Alphen measurements, the only adjustable parameters in the model are the phase of the oscil-

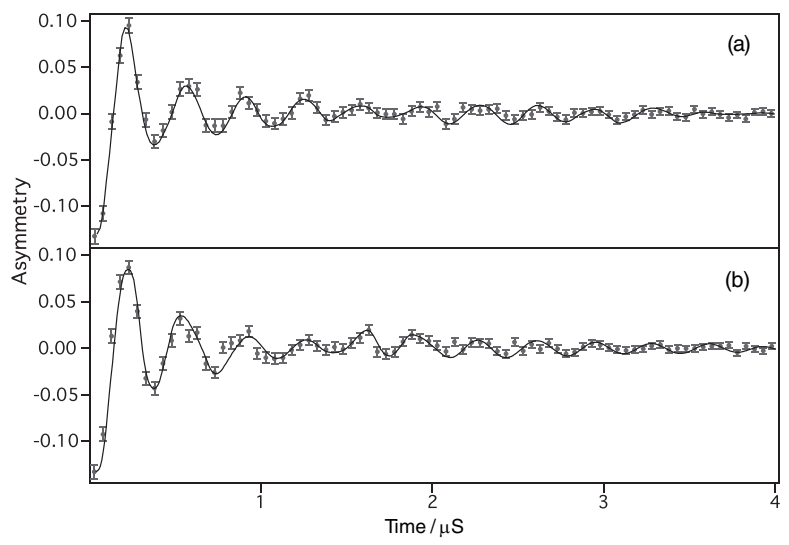

FIG. 2. The time evolution of the polarization of muons implanted into the sample with an energy of $10 \mathrm{keV}$, as revealed by the asymmetry in the number of detected positrons. The solid lines correspond to fits to the data using the same model as Figs. 3(a) and 3(b) (see text). The measurements were made in an applied field of $20 \mathrm{mT}$ in (a) the normal state, at a temperature of $10 \mathrm{~K}$ and (b) the superconducting state, at a temperature of $2.5 \mathrm{~K}$. 

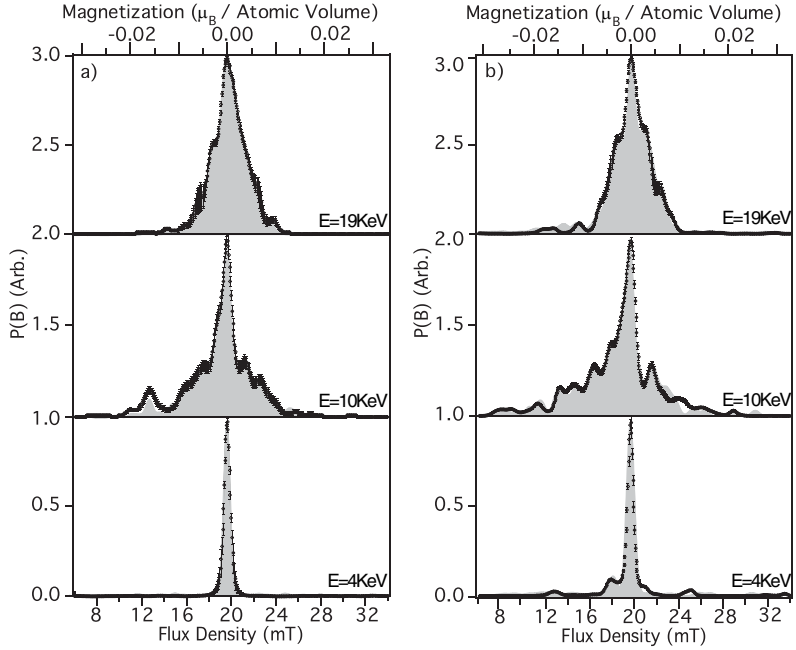

FIG. 3. The field probability distribution $p(B)$ for different implantation depths into the sample, revealing the presence of a spin-density wave inside the $\mathrm{Pb}$ layer (see text). The points and shaded areas correspond to the data and fit, respectively. It was measured in an applied field of $20 \mathrm{mT}$ in (a) the normal state, at a temperature of $10 \mathrm{~K}$ and (b) the superconducting state, at a temperature of $2.5 \mathrm{~K}$.

lation $\phi_{i}$, the amplitude $A_{i}$, and the exponent $n_{i}$. The phases of the two oscillations, $\phi_{1}$ and $\phi_{2}$, were found to be $3 \pi / 2 \pm 0.2$ and $\pi / 2 \pm 0.2$, respectively, and the exponent was found to be 0.86 (2) for both oscillations, which is surprisingly close to the value obtained for a similar $\mathrm{SDW}$ in a $\mathrm{Fe} / \mathrm{Ag} / \mathrm{Fe}$ trilayer [9]. The exponent $n_{i}$ is much smaller than for a simple RKKY model [3-5], although not dissimilar to that for theories of electron confinement in FM/N/FM trilayers [19]. The result is that the electronspin polarization decays relatively slowly in amplitude as a function of depth, and is thus evident even at our largest implantation energy.

We now turn to data taken at $T=2.5 \mathrm{~K}$, below the superconducting transition temperature, measured by field cooling immediately after the measurements were taken above $T_{c}$. Figure $3(\mathrm{~b})$ shows the $p(B) \mathrm{s}$ for the same energies under identical conditions as those measured above $T_{c}$. The resemblance of the raw data between Figs. 2(a) and 2(b) demonstrate that remarkably the SDW persists into the superconducting state, and this coexistence is also supported by the satellite peaks of Fig. 3(b). To understand the subtle differences that occur below $T_{c}$ we first turn to the field profile across a pure $\mathrm{Pb}$ thin film. We performed PNR measurements on a $220 \mathrm{~nm} \mathrm{~Pb}$ film, where we use the fact that the neutron spin is sensitive to the flux profile inside the sample. We used a nonmagnetic sample since PNR measurements to study the effects of superconductivity are not currently feasible on FM/S/FM trilayers. This is because the much stronger spin-dependent scattering from the magnetic layers masks the much weaker, subtle effects from the superconductor. The local nature of the
LE- $\mu$ SR technique avoids this difficulty and allows us to see effects inside the $\mathrm{Pb}$ film without interference from the Fe films. Fits to the PNR spin asymmetry [Fig. 4(b)] clearly indicate that the flux profile, given in Fig. 4(a), is rather different from that of a simple Meissner state [20]. Rather, the model shown comprises a highly concentrated row of vortices down the center of the sample, leading to a flux profile that exhibits flux expulsion near the surface and a maximum in the center, in the vicinity of the normal cores of the vortices $[21,22]$. The London penetration depth and Ginzburg-Landau coherence length were found to be $50(2) \mathrm{nm}$ and 55(2) nm respectively, with a vortex spacing of $450(10) \mathrm{nm}$. Fits to the LE- $\mu$ SR data, independent to the PNR fits, reveal a very similar flux profile inside the FM/S/FM trilayer, only with less flux expulsion and a slightly larger core size, as might be expected if the ferromagnetism acts to suppress the superconductivity. In order to increase the spin-dependent signal the PNR measurements were also taken at the somewhat higher field of $50 \mathrm{mT}$.

The presence of vortices alone, however, does not account for the $p(B)$ observed in the LE- $\mu$ SR data below $T_{c}$ [Fig. 3(b)]. In order to model successfully the field profile inside the $\mathrm{Pb} / \mathrm{Fe}$ film, one must also take into account the spatial phase of the oscillations. The model shown in Fig. 3(b) includes a $\pi / 2$ change in phase of one of the spin-density wave components compared to the value found in the normal state, such that $\phi_{1}$ and $\phi_{2}$ are $2 \pi \pm$ 0.2 and $\pi / 2 \pm 0.2$, respectively. The fits also require a $25 \%$ increase in amplitude for both oscillations to describe the data, suggesting an increase in the coupling of spins below the superconducting transition. To illustrate this further, Fig. 4(c) compares a set of data taken below $T_{c}$ using a model identical to that shown in Fig. 3(b), except that the phases were constrained to the values found above $T_{c}$. Thus it is clear that this $\pi / 2$ phase shift to the model provides a much better description of the frequency distribution below $T_{c}$, which is true for the data taken at all implantation energies [Fig. 3(b)]. For example, this phase change also reduces the local flux density in the vicinity of the $\mathrm{Fe} / \mathrm{Pb}$ interface, allowing a description of the additional satellites observed below $T_{c}$ at $4 \mathrm{keV}$ [Fig. 3(b)]. These are not visible above $T_{c}$ [Fig. 3(a)] because the muons in this region are rapidly depolarized outside of the time window.

While a number of previous experimental and theoretical studies have suggested the existence of LOFF-type states in the FM layers of hybrid multilayer systems [e.g., [7,8]], relatively little theoretical or experimental study has been carried out on the related effects inside the S layer. In a recent model calculation [6], it was found that spontaneous currents may flow near a superconductor/ FM interface. The currents in the FM flowed in the opposite direction to those in the superconductor, which died away exponentially on the scale of the FM coherence 


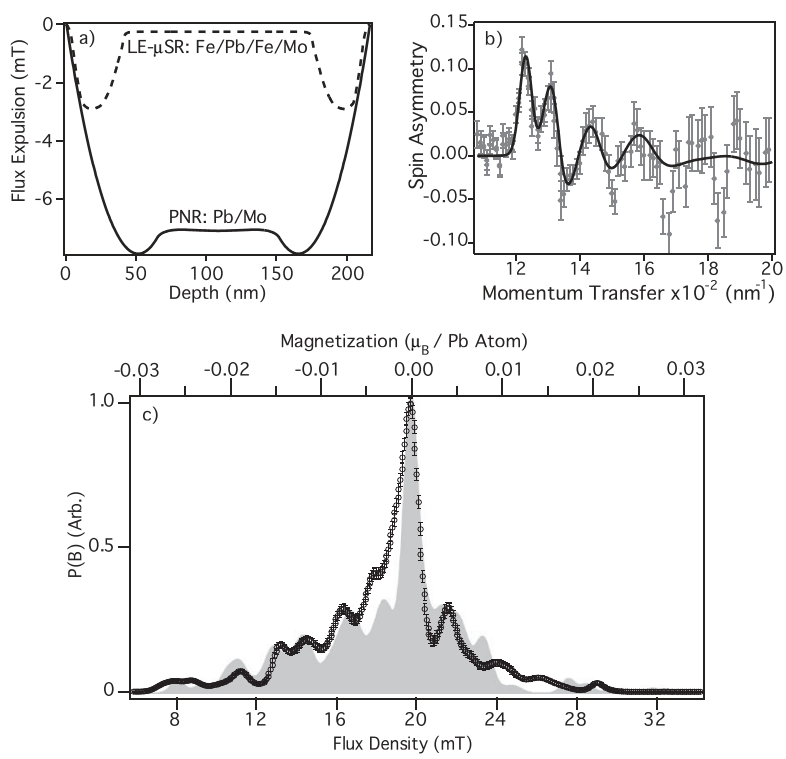

FIG. 4. (a) The field profile across a Pb film of thickness $220 \mathrm{~nm}$ in an applied field of $50 \mathrm{mT}$, deduced from a PNR experiment (solid). A similar profile is deduced from the fits to the LE- $\mu$ SR data below $T_{c}$ in the $\mathrm{Fe} / \mathrm{Pb} / \mathrm{Fe}$ trilayer at $20 \mathrm{mT}$ (dashed line). (b) The PNR spin asymmetry and fit to a model involving a single row of vortices in the middle of the film $[21,22]$. (c) One of the data sets shown in Fig. 2(b) is compared with an identical model, except that the phases were constrained to the values found above $T_{c}$.

length. The results presented in this Letter show a much longer range penetration of oscillatory spin polarization inside the $\mathrm{S}$ layer and it remains uncertain as to whether the LOFF mechanism is appropriate in this case.

It was proposed by Anderson and Suhl [23] that magnetic impurities present in a superconducting system could couple to the superconducting state. They suggested that the suppression of the spin susceptibility $\chi(q)$ around $q=$ 0 in the BCS superconducting state leads to a broad maximum in $\chi\left(q_{s}\right)$ at finite $q$. By analogy with the RKKY interaction this gives rise to a spatial variation of spin density characterized by a wave vector $q_{s} \sim$ $\left(3 \pi^{2} / 2 k_{F}^{2} \xi_{0}^{-1}\right)^{1 / 3}$. This mechanism has recently been discussed in the context of the magnetic superconductor $\mathrm{TmNi}_{2} \mathrm{~B}_{2} \mathrm{C}$, to explain the uncharacteristically long length scale for magnetic order observed in the superconducting state [24]. Substituting the values obtained from our fits gives $q_{s} \sim 3.2 \mathrm{~nm}^{-1}$, close to that of the first component of the SDW found in the normal state. This proximity to the natural wavelength for spin polarization may help explain why it is only this component of the SDW that appears to undergo a phase change in the superconducting state.

In conclusion, we have demonstrated the remarkable coexistence of a SDW with bulk superconductivity in the $\mathrm{S}$ layer in a FM/S/FM trilayer. Furthermore, the apparent enhancement of the SDW amplitude and $\pi / 2$ phase shift of one component of the SDW below $T_{c}$ indicate a profound coupling of these two forms of spin order. It remains, however, a challenge to theory to explain fully this persistence of the SDW and its accommodation to the onset of bulk superconductivity in these systems.

We thank S. Langridge, R. Dalgleish, and H.P. Weber for their support during the beamtime and T. M. Riseman for her version of the maximum entropy code. This work was performed at the Swiss Muon Source, Paul Scherrer Institute, Villigen, Switzerland and at ISIS, Rutherford Appleton Laboratory, Oxfordshire, UK.

*Electronic address: alanjohn.drew@physics.org

${ }^{\dagger}$ Also at Institut für Metallphysik und Nukleare Festkörperphysik, Technische Universität Braunschweig, D-38106 Braunschweig, Germany.

${ }^{\ddagger}$ Also at Physik Institut, Universität Zürich, CH-8057 Zürich, Switzerland.

${ }^{\S}$ Current Address PR/PRL Editorial Offices, One Research Road, Box 9000, Ridge, NY 11961-9000.

[1] P. Fulde and R. A. Ferrell, Phys. Rev. 135, A550 (1964).

[2] A. I. Larkin and Yu. N. Ovchinnikov, Zh. Eksp. Teor. Fiz. 47, 1136 (1964) [Sov. Phys. JETP 20, 762 (1965)].

[3] P. Bruno and C. Chappert, Phys. Rev. Lett. 67, 1602 (1991).

[4] K. Yosida, Phys. Rev. 106, 893 (1957).

[5] M. A. Ruderman and C. Kittel, Phys. Rev. 96, 99 (1954).

[6] M. Krawiec, B. L. Gyorffy, and J. F. Annett, Phys. Rev. B 66, 172505 (2002).

[7] M. G. Khusainov and Yu. N. Proshin, Phys. Rev. B 56, R14283 (1997).

[8] T. Kontos et al., Phys. Rev. Lett. 89, 137007 (2002).

[9] H. Luetkens et al., Phys. Rev. Lett. 91, 017204 (2003).

[10] E. Morenzoni et al., Physica (Amsterdam) 326B, 196 (2003).

[11] T. J. Jackson et al., Phys. Rev. Lett. 84, 4958 (2000).

[12] A. Suter et al., Phys. Rev. Lett. 92, 087001 (2004).

[13] S. J. Blundell and J. A. C. Bland, Phys. Rev. B 46, 3391 (1992).

[14] E. Morenzoni et al., Nucl. Instrum. Methods Phys. Res., Sect. B 192, 254 (2002).

[15] T. M. Riseman and E. M. Forgan, Physica (Amsterdam) 289B, 718 (2000).

[16] J. Skilling and R. K. Bryan, Mon. Not. R. Astron. Soc. 211, 111 (1984).

[17] A. V. Gold, Philos. Mag. 5, 70 (1960).

[18] A. V. Gold, Phil. Trans. R. Soc. A 251, 85 (1958).

[19] J. Mathon, A. Umerski, M. Villeret, and R. B. Muniz, Phys. Rev. B 59, 6344 (1999).

[20] H. Zhang et al., Phys. Rev. B 52, 10395 (1995).

[21] A. Drew et al. (to be published).

[22] S. W. Han et al., Phys. Rev. B 59, 14692 (1999).

[23] P. W. Anderson and H. Suhl, Phys. Rev. 116, 898 (1959).

[24] K. Norgaard et al., Phys. Rev. Lett. 84, 4982 (2000). 